\title{
Paclitaxel-induced Chest Pain and Left Bundle Branch Block in the Absence of Cardiac Ischemia
}

Jorge A Brenes-Salazar

Indian Journal of Critical Care Medicine (2019): 10.5005/jp-journals-10071-23281

\section{Case Description}

A 60-year-old woman with a history of hypertension, type II diabetes, and hyperlipidemia underwent her first cycle of intravenous chemotherapy with paclitaxel for endometrial clearcell adenocarcinoma at a standard rate of $135 \mathrm{mg} / \mathrm{m}^{2}$ over 3 hours (body surface area $2.10 \mathrm{~m}^{2}$ ).

Thirty minutes into her infusion, she complained of dizziness and heavy chest discomfort; her blood pressure was $105 / 59 \mathrm{~mm} \mathrm{Hg}$, a pulse of 75 beats per minute, and an oxygen saturation of $95 \%$ on ambient air; cardiopulmonary physical examination revealed reverse splitting of the second heart sound. An electrocardiogram was performed and showed a new, complete left bundle branch block (LBBB) (Fig. 1). Prior to the initiation of her infusion, her electrocardiogram displayed no conduction abnormalities at similar heart rates. Her symptoms spontaneously resolved within 10 minutes of stopping her infusion.

She was admitted to the cardiology service for additional workup. Her complete blood count and comprehensive metabolic
Department of Cardiovascular Diseases and Division of Geriatric Medicine, Mayo Clinic, Rochester, Minnesota, USA

Corresponding Author: Jorge A Brenes-Salazar, Department of Cardiovascular Diseases and Division of Geriatric Medicine, Mayo Clinic, Rochester, Minnesota, USA, Phone: +1-507-284-1644, e-mail: brenes-salazar.jorge@mayo.edu

How to cite this article: Brenes-Salazar JA. Paclitaxel-induced Chest Pain and Left Bundle Branch Block in the Absence of Cardiac Ischemia. IJCCM 2019;23(11):526-528.

Source of support: Nil

Conflict of interest: None

panel were within normal limits. Serial troponin I levels were obtained on admission, at 3-6 hours and were negative. A comprehensive echocardiogram was performed, which revealed normal left and right ventricular function, with no regional wall motion abnormalities. Coronary angiogram demonstrated no

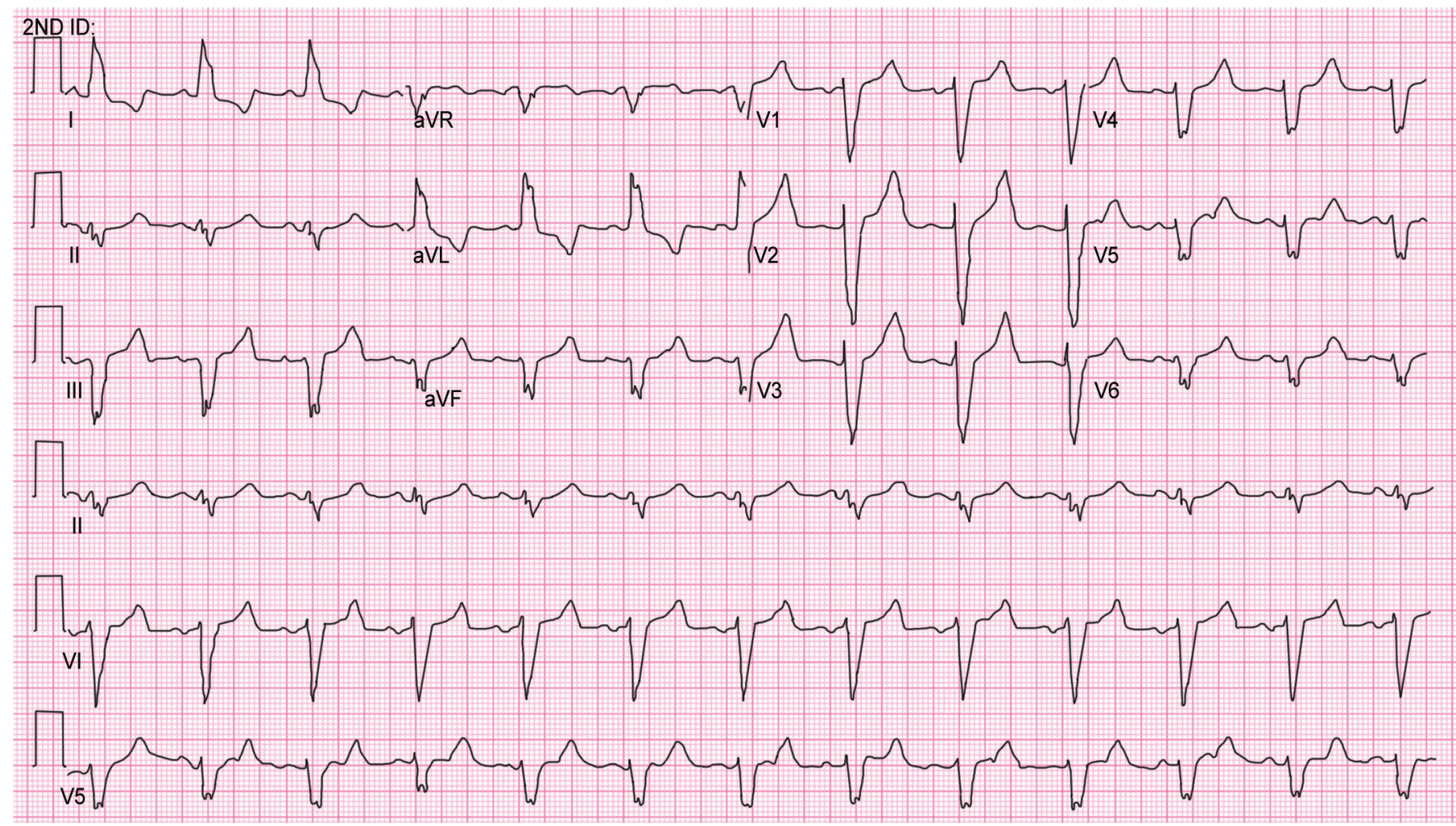

Fig. 1: Electrocardiogram obtained shortly after the onset of chest discomfort, which displays normal sinus rhythm at 75 beats per minute and a complete left bundle branch block, with ST and T wave abnormalities related to abnormal conduction

o The Author(s). 2019 Open Access This article is distributed under the terms of the Creative Commons Attribution 4.0 International License (https://creativecommons. org/licenses/by-nc/4.0/), which permits unrestricted use, distribution, and non-commercial reproduction in any medium, provided you give appropriate credit to the original author(s) and the source, provide a link to the Creative Commons license, and indicate if changes were made. The Creative Commons Public Domain Dedication waiver (http://creativecommons.org/publicdomain/zero/1.0/) applies to the data made available in this article, unless otherwise stated. 


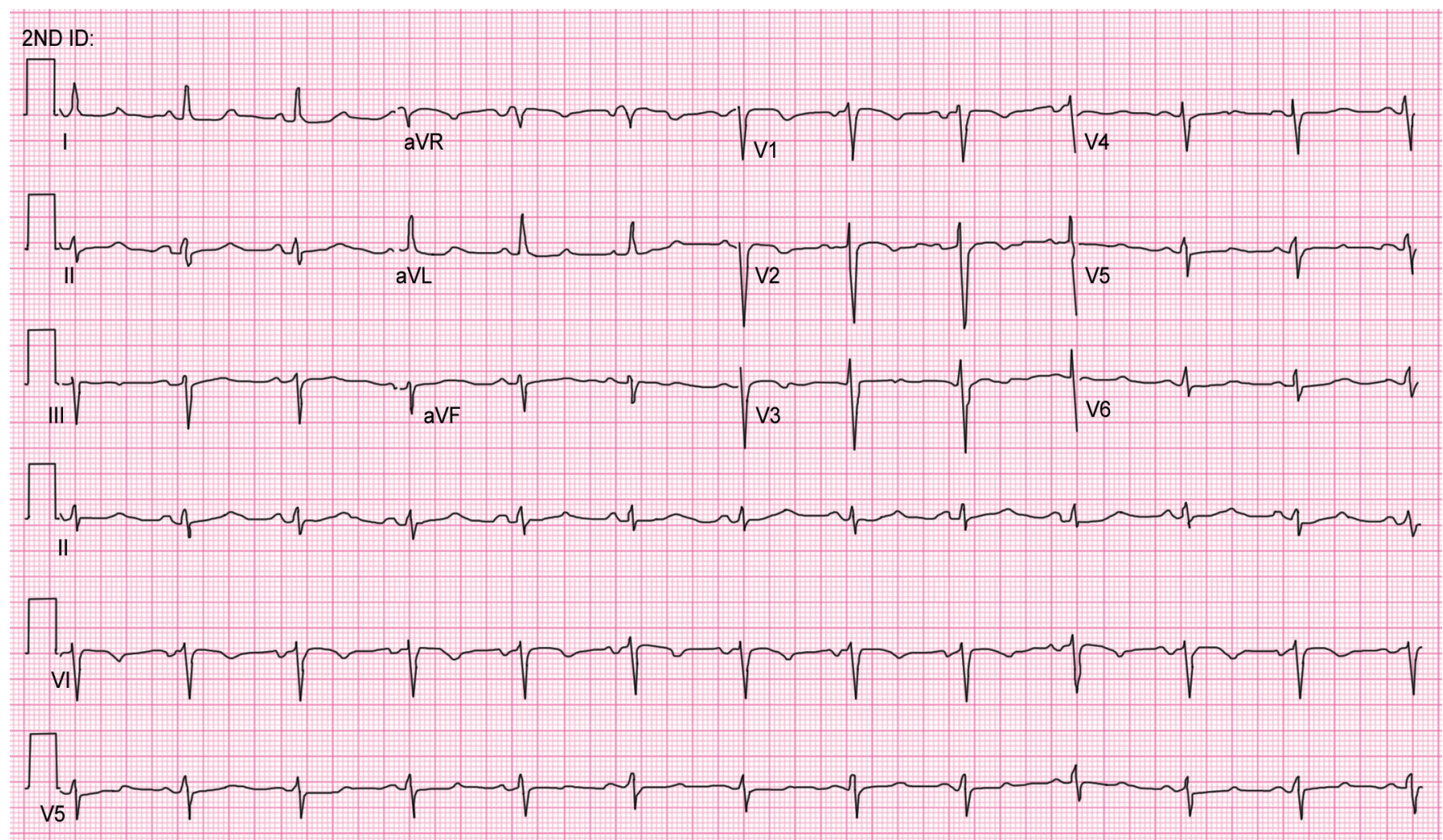

Fig. 2: Electrocardiogram obtained 1 week after hospital discharge, which demonstrates resolution of left bundle branch block; heart rate is 73 beats per minute

Table 1: Acute/subacute causes of left bundle branch block

\begin{tabular}{ll}
\hline Etiology & Diagnostic clues \\
\hline Acute coronary syndromes (e.g., anterior & Sgarbossa's criteria, elevated troponins, established coronary artery disease or multiple risk \\
STEMI) & factors \\
Myocarditis & Heart failure, elevated inflammatory markers, elevated troponins \\
Hyperkalemia & Rare, usually rapid rise in serum potassium \\
Toxic & $\begin{array}{l}\text { History of recent exposure to heavy metals, cocaine, chemotherapy, antiarrhythmics } \\
\text { (particularly class I) }\end{array}$ \\
Infectious & Specific presentations of Lyme disease, Chagas disease, diphtheria, acute rheumatic fever, etc. \\
Rate-related (functional) & Transient, several long R-R intervals followed by short R-R cycles, disappears at lower heart \\
& rates \\
\hline
\end{tabular}

obstructive coronary disease. Her oncologist was informed of the results, and an alternative chemotherapy regimen was chosen. At 1-week follow-up, she reported no recurrence of symptoms; repeat electrocardiogram displayed resolution of her LBBB (Fig. 2).

LBBB results when the normal activation sequence in the HisPurkinje system is interrupted, with asynchronous and delayed activation of the left ventricle. ${ }^{1}$ In most cases, LBBB is associated with underlying conduction system disease, which can be progressive. Multiple chronic conditions that promote myocardial fibrosis, such as hypertension, atherosclerosis, and valvular disease, can contribute to the gradual development of LBBB. However, there are some clinical scenarios, with acute coronary syndromes being the most commonly invoked, in which LBBB presents in a rather acute or subacute manner, leading to different diagnostic considerations (Table 1).

Paclitaxel is a natural product, originally isolated from the Pacific yew tree (Taxus brevipholia) that exhibits antineoplastic activity by inhibition of tubular polymerization. In early clinical trials, major hypersensitivity reactions were reported with the use of paclitaxel; ${ }^{2}$ such reactions led to routine telemetry monitoring during subsequent phases, which unveiled previously unnoticed cardiovascular manifestations. Most of these are related to asymptomatic sinus tachycardia. ${ }^{3}$ More serious cardiovascular events are rare but include hypotension, varying degrees of atrioventricular (AV) block, ventricular tachycardia, and acute coronary syndromes. ${ }^{4,5}$ Left bundle branch block has been reported with a frequency of $4 \%{ }^{2}$

From a clinical standpoint, symptoms such as wheezing, dizziness, and rash have been described in the setting of hypersensitivity reactions to paclitaxel, but chest pain has been reported only in rare occasions. ${ }^{6}$ The concomitant presence of chest discomfort and a new LBBB with the use of paclitaxel has not been previously described in the literature. Such presentation raised suspicion of an acute coronary syndrome and hence motivated the transfer of care of our patient.

The mechanism by which paclitaxel induces LBBB is not entirely clear, but the absence of other dynamic ischemic changes on the electrocardiogram and most importantly no evidence of myocardial 
injury as measured by troponin levels, which very strongly suggests a nonischemic process, as myocardial contractility is not significantly dependent on microtubular function. Conversely, the sarcoplasmic and sarcotubular systems have important roles in electrical impulse conduction in the sinoatrial and atrioventricular nodes, as well as the His-Purkinje system, ${ }^{7}$ which would be consistent with the wide array of electrocardiographic disturbances reported with the use of paclitaxel.

Fortunately, most of the electrocardiographic disturbances associated with paclitaxel appear to be asymptomatic, transient, and tend to resolve spontaneously after discontinuation of the agent. Nevertheless, they often recur when the patient is rechallenged with the drug and therefore may prompt for alternative chemotherapy choices, particularly with presentations such as high-grade AV block or ventricular tachycardia.

\section{References}

1. Hecht HH, Kossmann CE, Childers RW, Langendorf R, Lev M, Rosen $\mathrm{KM}$, et al. Atrioventricular and intraventricular conduction. Revised nomenclature and concepts. Am J Cardiol 1973;31(2):232-244. DOI: 10.1016/0002-9149(73)91036-9.
2. Kamineni P, Prakasa K, Hasan SP, Akula R, Dawkins F. Cardiotoxicities of paclitaxel in African Americans. J Natl Med Assoc 2003;95(10):977-981.

3. Trimble EL, Adams JD, Vena D, Hawkins MJ, Friedman MA, Fisherman JS, et al. Paclitaxel for platinum-refractory ovarian cancer: results from the first 1,000 patients registered to National Cancer Institute Treatment Referral Center 9103. J Clin Oncol 1993;11(12):2405-2410. DOI: 10.1200/JCO.1993.11.12.2405.

4. Yu AF, Manrique C, Pun S, Liu JE, Mara E, Fleisher M, et al. Cardiac safety of paclitaxel plus trastuzumab and pertuzumab in patients with HER2-positive metastatic breast cancer. Oncologist 2016;21(4): 418-424. DOI: 10.1634/theoncologist.2015-0321.

5. Shah K, Gupta S, Ghosh J, Bajpai J, Maheshwari A. Acute non-ST elevation myocardial infarction following paclitaxel administration for ovarian carcinoma: a case report and review of literature. J Cancer Res Ther 2012;8(8):442-444. DOI: 10.4103/0973-1482. 103530.

6. Rowinsky EK, McGuire WP, Guarnieri T, Fisherman JS, Christian MC, Donehower RC. Cardiac disturbances during the administration of taxol. J Clin Oncol 1991;9(9):1704-1712. DOI: 10.1200/JCO.1991.9. 9.1704 .

7. Anderson $\mathrm{RH}$, Becker $\mathrm{AE}$, Tranum-Jensen, Janse MJ. Anatomicroelectrophsiological correlations in the conduction system: a review. Br Heart J 1981;45(1):67-82. DOI: 10.1136/hrt.45.1.67. 